\title{
A Mathematical Model for the Flow Resistance and the Related Hydrodynamic Dispersion Induced by River Dunes
}

\author{
Marilena Pannone, Annamaria De Vincenzo, and Francesco Brancati \\ School of Engineering, University of Basilicata, Viale dell'Ateneo Lucano 10, 85100 Potenza, Italy \\ Correspondence should be addressed to Marilena Pannone; marilena.pannone@unibas.it
}

Received 5 July 2013; Revised 1 October 2013; Accepted 1 October 2013

Academic Editor: Mohamed Fathy El-Amin

Copyright ( 2013 Marilena Pannone et al. This is an open access article distributed under the Creative Commons Attribution License, which permits unrestricted use, distribution, and reproduction in any medium, provided the original work is properly cited.

\begin{abstract}
Present work is aimed at the derivation of a simply usable equation for the total flow resistance associated with river bedforms, by a unifying approach allowing for bypassing some of the limiting restrictions usually adopted in similar types of studies. Specifically, we focused on the effect induced by the out-of-phase free surface undulations appearing in presence of sand dunes. The proposed expression, obtained by combining the balance of momentum referred to the control volume whose longitudinal dimension coincides with the dune wavelength and the energy balance integrated between its extreme sections, was tested by comparison with some laboratory experimental measurements available in the literature and referred to steady flow past fixed, variably rough bedforms. In terms of shear stress or friction factor, the proposed theory provides estimates in good agreement with the real data, especially if evaluated against the performances provided by other classical similar approaches. Moreover, when analyzed in terms of hydrodynamic dispersive properties as a function of the skin roughness on the basis of a previously derived analytical solution, the dune-covered beds seem to behave like meandering channels, responsible for a globally enhanced fluid particles longitudinal spreading, with a relatively reduced effect in the presence of less pronounced riverbed modelling.
\end{abstract}

\section{Introduction}

Stream flow along undulated beds has been widely studied in the past, both analytically and experimentally, in terms of resistance factors [1-12]. Several more recent experimentalanalytical works have dealt with the fluid-dynamical aspect of the problem through innovative techniques (e.g., [13-16]).

Indeed, for a long time it has been commonly accepted that large-scale bedforms like dunes and bars are generated by large-scale turbulence, vertical or horizontal, respectively [17-20] through the so-called bursting processes [19-26]. Bursting process, or simply burst, is synonym of evolution of a large macroturbulent eddy. In the zone of highest resistance, that is, near the flow solid boundaries, very large values of fluctuating shear stresses make the fluid rolling-up into eddies. The so-generated vortices move away from river bed or banks as they are conveyed downstream by the flow, in the meantime diffusing, coalescing, eventually breaking-up at the borders of the domain, and thus generating a spacetime rhythmic sequence [27].

The periodic (or, more realistically, pseudoperiodic) distortion of the velocity field, in its turn, triggers off the deformation of the mobile bed and, therefore, the creation of typical bedforms. From turbulence measurements, it has been found that the fully-developed dune wavelength $\Lambda$ scales with the average flow depth $H: \Lambda \sim 6 H$. Unavoidably these bedforms, which constitute the physical imprint of the turbulent flow, are affected by the strong and ubiquitous random element characterizing the high Reynolds number processes and should be therefore analyzed by resorting to suitable stochastic approaches (e.g., [28-31]). In any case, even leaving aside the random aspect, in turbulent flow conditions and in presence of solid transport, there is a wide set of elements from whose interaction the total resistance opposed by the river bed to the water flux is determined. For instance, the skin roughness, commonly identified with 
shape and dimensions of the material constituting the wetted contour, can incorporate other factors whose quantitative effect can not be evaluated with the same accuracy, that is, the concentration and the distribution of the grains, or the vegetation. The latter, which is usually present both on the bottom and the banks of the channel, is associated with a sometimes considerable reduction of the bed capacity, slowing down the flow in a more or less pronounced fashion depending on type, height, density, and distribution of the arboreal species. To the skin roughness, it is then necessary to add the resistance induced by the geometric bed irregularities, like those represented by particularly uneven wetted contours and, obviously, those deriving from the continuously evolving bed configuration and related to the specific type of solid load (ripples, dunes, and anti-dunes up to a limit grain size and macroroughness for larger grains dimensions). Furthermore, one should account for the dynamical influence of the solid transport itself, either as bed or suspended load, which tends to highlight the effect of the bed irregularities and to increase the flow resistance and, finally, for the changes that the transported solid particles cause on the physical-chemical characteristics of the stream. Even restricting the investigation field to the uniform or on average-uniform flows, and therefore neglecting the contributions to the resistance represented by obstacles and obstructions (i.e., bridge piers, dams and alluvial fans), as well as those attributable to the flow rate oscillations, it is clearly difficult to quantify the effects due to so many factors.

The aim of present work is represented by the analytical derivation of a closed-form solution for the estimation of the total resistance in presence of river dunes (a typically two-dimensional sand bed pattern) in perfect equilibrium conditions, that is, neglecting the effects of their downstream migration, based on the elaboration of the global momentum and energy balances. The final expression accounts for skin and shape roughness in a nontrivially additive way, and for the free surface undulation as a function of Froude number; the intrinsically nonstationary phenomenon of the flow separation near the dune crest is evaluated in terms of resistance resorting to an equivalent steady flow configuration.

The proposed approach is then tested by comparing the analytical estimates of the total friction factor with the laboratory measurements provided by Engel and Lau [9], and the analytical estimates of the total shear stress with the laboratory measurements documented in McLean et al. [15]. Moreover, based on a previously derived closed-form solution for the hydrodynamic dispersion coefficient [31], the data by Engel and Lau [9] are utilized to investigate the relationship between the flow resistance and the dispersive properties of streams flowing past undulated sand beds.

\section{Mathematical Formulation}

Consider steady and periodically uniform two-dimensional streams. For weak curvatures of the fluid particles trajectory determined by bottom and free surface undulations, the pressure can be assumed as hydrostatically distributed along all the verticals. Note that, in the separated flow zone

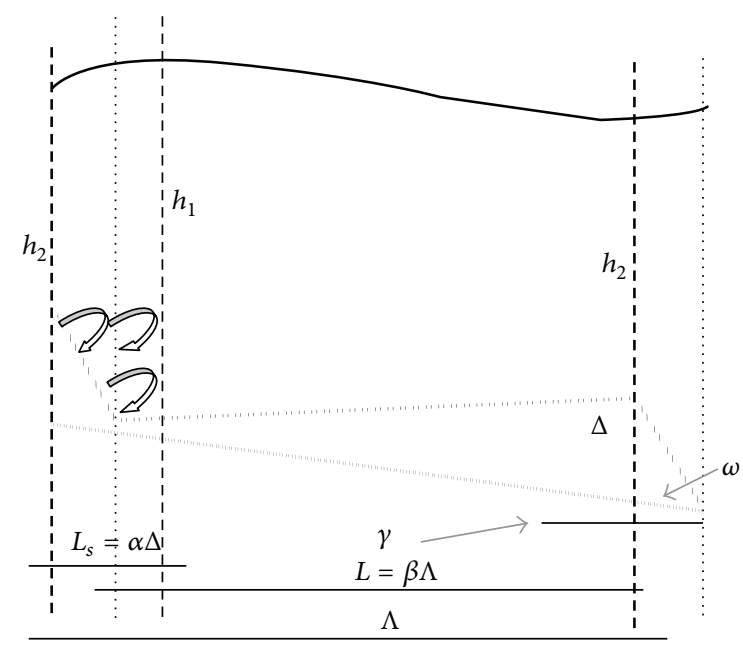

FIGURE 1: Sketch of the physical model.

downstream the dune crest, the validity of the assumption is guaranteed by Helmholtz theory and by the substantial immobility of the fluid subject to the geopotential. In the same zone, the shear stresses are usually neglected due to their very small negative values. Finally, in the low Froude number regime flows, water and bed sediment waves are commonly out of phase. Specifically, it is reasonable to expect the maximum drop of the free surface near the dune peak, due to the acceleration induced by its upstream positive slope, and the maximum rise in the separated and highly turbulent zone where the flow, after having achieved the minimum velocity, realigns and accelerates again (see Figure 1). In the most standard situations, with the basic geometric parameters assuming the most common values, the separation length $L_{s}$ ranges from about $4 \Delta$ to about $6 \Delta$, where $\Delta$ indicates the dune height measured along the vertical [9]. Generally, $L_{s}=\alpha \Delta$. In order to estimate the total friction factor in such circumstances, we have applied the momentum balance equation to the control volume (basically, the flow domain identified by the dunes sequence wavelength) delimited in Figure 1 by the free surface, the dune contour, and the vertical bold dashed lines:

$$
\mathbf{I}+\mathbf{M}_{o}-\mathbf{M}_{i}=\mathbf{G}+\mathbf{\Pi}
$$

where $\mathbf{I}$ indicates the local inertia, $\mathbf{M}_{o}$ and $\mathbf{M}_{i}$ the outflow and inflow momentum flux, respectively, $\mathbf{G}$ the control volume weight and $\Pi$ the resultant of the applied surface forces (isotropic pressures plus shear stresses). In turbulent steady conditions, and because of the periodicity of the peculiar velocity field, the projection of that equation along the horizontal direction $x$ yields

$$
\Pi_{x}=\tau^{\prime} L+p_{b}\left(\Delta-i_{f} L\right)-p_{s}\left[\Delta+i_{f}(\Lambda-L)\right]=0
$$

with

$\tau^{\prime}$ : shear stress due to the skin roughness only;

$\Lambda$ : horizontal length of the sand wave; 
$L=\beta \Lambda=\{1-\Delta / \Lambda[\cos (\omega+\gamma) \cos \gamma / \sin \omega]\} \Lambda:$ horizontal length of the dune back;

$i_{f}=\tan \gamma:$ bottom slope (generally weak);

$\omega$ : angle of the downstream slope;

$p_{b}$ : average pressure acting on the dune back;

$p_{s}$ : average pressure acting on the dune in the separation zone $\left(L_{s}\right)$.

It is worth noting that in (2) the length of the control volume where the skin shear stress is considered active coincides with the whole upstream dune surface. That assumption is justified for bed disturbances characterized by relatively small amplitude (small $L_{s}$ ). Considering an approximately linear variation of the free surface profile associated with not exceedingly steep bedforms, and $\omega \rightarrow \pi / 2$, we obtain

$$
p_{b}=\gamma_{w} H \quad p_{s} \cong \gamma_{w}\left(H-\frac{\delta}{2}\right),
$$

where $\delta=h_{1}-\Delta-h_{2}$ represents the difference between maximum and minimum free surface elevation and $\gamma_{w}$ is the water specific weight. Actually, the second of the previous identities should be rewritten as:

$$
\begin{gathered}
p_{s}=\gamma_{w}(H-b), \\
b=\frac{\delta}{2}\left[\frac{\beta}{1-\alpha(\Delta / \Lambda)}-\frac{(1-\beta)}{\alpha(\Delta / \Lambda)}\right] .
\end{gathered}
$$

However, (4) tends to its approximate counterpart for small $\Delta / \Lambda$ and $\omega \rightarrow \pi / 2$. The relationship linking $\delta$ to the flow kinematic parameters can be derived by considering the energy balance ( $1 D$ Saint Venant equation) integrated between Sections 1 and 2, characterized by maximum and minimum flow depths $h_{1}$ and $h_{2}$, respectively. Under the above-mentioned conditions,

$$
i_{f} L+h_{1}+\frac{U_{1}^{2}}{2 g}-\frac{\tau^{\prime} L}{\gamma_{w} H}=\Delta+h_{2}+\frac{U_{2}^{2}}{2 g}
$$

with $U_{1}$ and $U_{2}$ indicating the inflow and outflow section averaged velocities, respectively, and $\tau^{\prime} L / \gamma_{w} H$ the unit weight energy loss. The result reads

$$
\delta=\frac{U_{2}^{2}-U_{1}^{2}}{2 g}-\left(i_{f}-\frac{\tau^{\prime}}{\gamma_{w} H}\right) L .
$$

Now, by virtue of continuity: $U H=U_{1} h_{1}=U_{2} h_{2}$, and assuming $H$ as the geometric mean of the extreme flow depth values: $H \sim\left(h_{1} h_{2}\right)^{1 / 2}$, one obtains

$$
\delta=\frac{\Delta F_{r}^{2}}{1-F_{r}^{2}}-\left(i_{f}-\frac{\tau^{\prime}}{\gamma_{w} H}\right) \frac{L}{1-F_{r}^{2}},
$$

where $F_{r}=U /(g H)^{1 / 2}$ indicates the average Froude number (kinetic index). Finally, the combination of (2), (3) and (7) yields the following expression for the total shear stress $\tau=\gamma H i_{f}$ as a function of Froude number and geometric bedforms parameters:

$$
\begin{aligned}
\tau= & \beta\left\{1+\frac{\Lambda}{2 H\left(1-F_{r}^{2}\right)}\left[\frac{\Delta}{\Lambda}+(1-\beta) i_{f}\right]\right\} \tau^{\prime} \\
& +\frac{\gamma_{w}}{2}\left[\frac{\Delta}{\Lambda}+(1-\beta) i_{f}\right]\left(\frac{\Delta F_{r}^{2}}{1-F_{r}^{2}}-\frac{\beta \Lambda i_{f}}{1-F_{r}^{2}}\right) .
\end{aligned}
$$

Equation (8) can straightforwardly be transformed in terms of friction factors invoking the well-known relationships:

$$
f=\frac{8 \tau}{\rho U^{2}} \quad f^{\prime}=\frac{8 \tau^{\prime}}{\rho U^{2}}
$$

where $\rho$ is the water density, leading to

$$
\begin{gathered}
f=A f^{\prime}+B, \\
A=\beta\left\{1+\frac{\Lambda}{2 H\left(1-F_{r}^{2}\right)}\left[\frac{\Delta}{\Lambda}+(1-\beta) i_{f}\right]\right\}, \\
B=4\left[\frac{\Delta}{\Lambda} \frac{1}{1-F_{r}^{2}}-\frac{\Lambda}{H} \frac{\beta i_{f}}{F_{r}^{2}\left(1-F_{r}^{2}\right)}\right] .
\end{gathered}
$$

As (10) clearly suggests, the proposed approach produces an analytical expression for the total friction factor which is not the mere superposition of the effects of skin roughness and bed modelling, as it happens in all the previous works dealing with the same topic by analytical tools. On the contrary, besides a term dependent on the bed geometric parameters and on the Froude number only $(B)$, which essentially accounts for the contribution to the flow resistance coming from the macroscopic bed deformation, it includes a coefficient $(A)$ which modulates the skin friction factor $f^{\prime}$ on the basis of the same characteristics and, therefore, accounts for the declining contribution of the grain roughness when the bottom undergoes a transition from flat to mildly steep. The derivation of the hydrodynamic dispersion coefficient in presence of sand dunes sequences was carried out based on a stochastic Lagrangian model [31], extensively discussed in the next section, when the corresponding solution is analyzed in terms of dependence on the dunes flow resistance.

\section{Results and Discussion}

In order to test the proposed formulation, we employed the experimental data provided by Engel and Lau [9], because they cover, in our opinion, a rather meaningful and well controlled range of geometrical and hydrodynamical parameters for approximately two-dimensional flow in presence of dunes. In their laboratory surveys, the authors utilized artificial and fixed bedforms characterized by a constant height $\Delta$ equal to $3 \mathrm{~cm}$, a variable slope $\Delta / \Lambda$ ranging from 0.02 to 0.07 , and a downstream angle $\omega=30^{\circ}$. The skin roughness was made by a fixed grain layer with a $D_{50}$ varying between 0.35 and $9 \mathrm{~mm}$. Furthermore, based on what was recommended by Yalin [4] and others, flow conditions and 


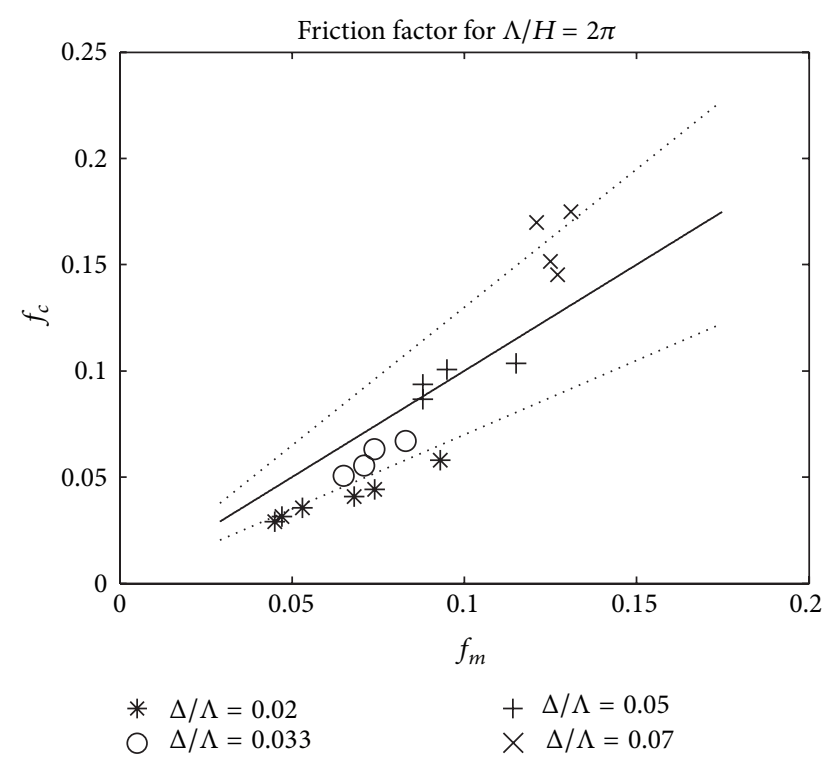

FIGURE 2: Comparison between measured [9] and calculated friction factors. The dotted lines correspond to the $70 \%$ confidence interval.

effects were analyzed for ratios of wavelength to average depth $\Lambda / H$ equal to $2 \pi$ (which is almost 6 ) and 5 ; the average bed slope $i_{f}$ and the Froude number Fr varied in a nonsystematic fashion, from $4.16 \times 10^{-4}$ to 0.022 and from 0.22 to 0.42 , respectively. Finally, the estimation of the skin friction factor was carried out based on the following logarithmic formula:

$$
f^{\prime}=1.28\left(\ln \frac{11 H}{K_{s}}\right)^{-2},
$$

where $K_{s}$, sand-equivalent roughness index, can be approximated by $2 D_{50}$. Figures 2 and 3 illustrate, for $\Lambda / H$ equal to $2 \pi$ and 5 , respectively, the comparison between the values of the total friction factor experimentally measured and those mathematically obtained through the combination of momentum and energy balances (10). As already mentioned, for each of the subcategories representing the different dunes slopes, $i_{f}$ and Fr vary in a non systematic fashion, while the relative roughness $D_{50} / H$ increases along with both the calculated and the measured friction factors, ranging from 0.0016 to 0.0513 for $\Lambda / H=2 \pi$ and from 0.0013 to 0.0402 for $\Lambda / H=5$. The grain Reynolds number $u_{*} K_{s} / \nu$, with $u_{*}$ indicating the shear velocity and $v$ the kinematic water viscosity, is always larger than 70, conventionally meaning rough fully turbulent flow.

As Figures 2 and 3 (which display the 70\% confidence intervals also) clearly show, the best agreement is obtained for $\Delta / \Lambda=0.05$, that is, for mildly steep dunes. Conversely, the theoretical formula underestimates the total friction factor for the flatter bedforms $(\Delta / \Lambda=0.02$ e 0.033$)$ and overestimates that for the steeper ones $(\Delta / \Lambda=0.07)$. Given the hypotheses at the basis of (10), it is reasonable to expect a deterioration of the performance of the equation as the dune slope increases, whereas it is worth discussing the discrepancy detected in the field of the flatter bedforms.

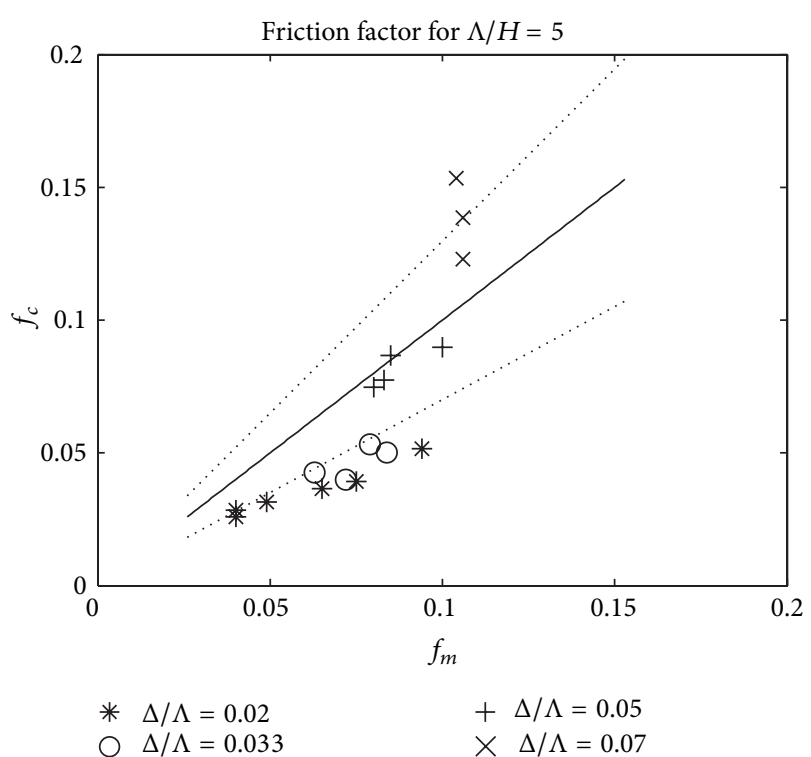

Figure 3: Comparison between measured [9] and calculated friction factors. The dotted lines correspond to the $70 \%$ confidence interval.

Indeed, it is possible that the proposed formula is not able to completely account for the greater or lesser relevance to be attributed to the skin roughness as a function of the dune slope and, although it is not a simple effects superposition, it should perhaps incorporate a modulation coefficient of $f^{\prime}$ which could vary with the magnitude of the flow dragging action and, presumably, in a nonlinear way with the dune slope. In any case, considering the possibility of measurement errors, the assumptions underlying the closed form solution (which, for instance, unavoidably neglects the pulsatile and crucial phenomenon of the vortex shedding at the dune crest), and a percentage error which is on average smaller than 20-25\%, we can conclude that (10) incorporates within a reasonable degree of approximation the influence of bottom and free surface undulations on the flow resistance in riverbeds. On the other hand, the comparison with the performances provided by other compact expressions for the total $f$ (Engelund [5]; Garde and Ranga Raju [6]; Vanoni and Hwang [7], resp.):

$$
\begin{gathered}
f=f^{\prime}+4 \frac{\Delta}{\Lambda} \frac{\Delta}{H}, \\
f=\frac{8}{\left[3.2\left(H / D_{50}\right)^{1 / 6}\right]^{2}}, \\
f=\frac{8}{\left(9.3 \log \left(\Lambda H / \Delta^{2}\right)-6.5\right)^{2}}
\end{gathered}
$$

effectively demonstrates (see Figure 4, displaying the histograms of the percentage errors for the 4 available equations, including (10)) the interpretative improvement represented by the present study. 
Figure 5 shows the behaviour of (10) as a function of Froude for $\Lambda / H=2 \pi, i_{f}=0.0012, H=0.17 \mathrm{~m}, D_{50}=$ $0.0005 \mathrm{~m}$, and the 4 sample values of $\Delta / \Lambda$. Note that the selected range of Froude corresponds to the interval of values detected in the Engel and Lau experiments, while the selected values of bed slope, depth, and grain size have been obtained by averaging the laboratory data. The estimates of $f$ are obviously increasing, Fr being the same, as $\Delta / \Lambda$ increases. The variation of $f$ with $\mathrm{Fr}$ is not too pronounced for weak dune slopes, and that is in agreement with the conclusions drawn by Engel and Lau [9] themselves, which were nevertheless based on measurements carried out by letting Fr vary in a non systematic way. The substantial invariance with respect to the kinetic flow index does not hold for the steeper bedforms. Indeed, with $\Delta / \Lambda=0.07$ the total $f$ ranges from about 0.12 for $\mathrm{Fr}=0.24$ to about 0.16 for $\mathrm{Fr}=0.45$. The effect of the increasing average flow velocity on the rough bedforms-induced acceleration is further highlighted in Figure 6, showing the relative free surface drop $\delta / \Delta$ (with $\delta$ given by (7)) as a function of the Froude number for $\Lambda / H=$ $2 \pi$. The diagram seems to detect the existence of a single mathematical relationship linking $\delta / \Delta$ to Fr regardless of the dune slope, with values up to the $18 \%$ for the faster streams.

In order to double-check the soundness of the proposed approach, (8) was tested based on the experimental measurements provided by McLean et al. [15]. In that data series (in all 8 runs) the selected dune slopes were equal to 0.05 (runs $2,3, \mathrm{~T} 5(7)$, and $\mathrm{T} 6(8)$ ) or 0.1 (runs 4, 5, 6, and 7). The ratio of wavelength to average flow depth was 20 or 10 , respectively (thus, larger than what is considered the "expected" value $(\sim 6)$ of that parameter). Froude number ranged from 0.1 to 0.31 . The measured quantities included the total shear stress estimated from the average flow depth $\gamma_{w} H i_{f}$, the total shear stress estimated from the hydraulic ratio $\gamma_{w} R i_{f}$, and the total shear stress obtained at the bottom from the Reynolds formula $-\rho\langle u w\rangle_{0}$, with $u$ and $w$ indicating the fluctuating component of longitudinal and vertical velocity, respectively, and the angle brackets indicating the ensemble mean. Note that, for runs from 2 to 7 , with a channel width equal to $0.9 \mathrm{~m}$, the percentage error in substituting the actual hydraulic radius with the average flow depth $[(R-H) / R] \%$ was $-35.11 \%$, $-121.33 \%,-35.33 \%,-35.33 \%,-66.67 \%$, and $-124.44 \%$. Thus, the experimental setup reproduced a situation considerably far from the two-dimensional flow assumed in our mathematical formulation. As Figure 7 clearly shows, although onehalf of the runs was performed with a very high (almost "extreme") value of dune slope (0.1) and the boundary effect was definitely not negligible, the shear stress estimates based on (8) (black asterisks) fall well inside the range of $\tau$ values identified by the experimental measurements or, at the most, coincide with one/two of them, further demonstrating the robustness of present first-order analytical approach.

Finally, for the sake of completeness, Figure 8 shows the behaviour of the dimensionless hydrodynamic dispersion coefficient as a function of the skin friction factor for the four different dune slopes whose effects were analyzed in the present work. The dimensionless coefficient $D_{x}$ was evaluated on the basis of the analytical solution proposed by Pannone
[31], starting from the Lagrangian stochastic interpretation of the fluid particles spreading process:

$$
D_{x}(t)=\frac{1}{2} \frac{d X_{11}}{d t}=\int_{0}^{t}\left\langle U^{\prime}[X(t)] U^{\prime}[X(\tau)]\right\rangle d \tau,
$$

where the primes indicate the random fluctuations, $X=X(t)$ is the longitudinal particle trajectory, $X_{11}(t)=\left\langle X^{\prime}(t)^{2}\right\rangle$ is the particle trajectory covariance, and $\left\langle U^{\prime}[X(t)] U^{\prime}[X(\tau)]\right\rangle$ the Lagrangian velocity covariance. Incidentally, the (periodic) random nature of the Lagrangian velocity field derives from the sampling of the section averaged velocity distribution associated with the sand waves sequence (and then represented by a strong deterministic and periodic trend), by fluid particles subject to diffusive transport mechanisms usually described by Fick's law and Brownian paths. Thus, assuming a bed elevation covariance represented by a single cosine function, with a variance proportional to the dune height squared (e.g., [27]):

$$
\langle\widetilde{h}(x) \widetilde{h}(x+\xi)\rangle=C_{h}(\xi)=\left(\frac{\Delta}{2}\right)^{2} \cos \left(\frac{2 \pi \xi}{\Lambda}\right)
$$

and relying on the continuity condition for not exceedingly high and steep dunes, that is, for relatively small depth deviations $\widetilde{h}$ :

$$
U(x)=\frac{U H}{h(x)}=\frac{U H}{H+\widetilde{h}(x)} \cong U\left(1-\frac{\widetilde{h}(x)}{H}\right),
$$

the following first order solution for small $\Delta / \Lambda$ was obtained [31]:

$$
\begin{aligned}
D_{x}(t)= & \frac{\left(\Delta^{2} / 16\right)(U / H)^{2}}{\left(\pi^{2} / \Lambda^{2}\right)\left(\left(4 \pi^{2} \varepsilon_{x}^{2} / \Lambda^{2}\right)+U^{2}\right)} \\
& \times\left\{\frac{4 \pi^{2} \varepsilon_{x}}{\Lambda^{2}}\left[1-\exp \left(-\frac{4 \pi^{2} \varepsilon_{x}}{\Lambda^{2}} t\right) \cos \left(\frac{2 \pi U}{\Lambda} t\right)\right]\right. \\
& \left.+\frac{2 \pi U}{\Lambda} \exp \left(-\frac{4 \pi^{2} \varepsilon_{x}}{\Lambda^{2}} t\right) \sin \left(\frac{2 \pi U}{\Lambda} t\right)\right\} .
\end{aligned}
$$

With $D_{x}=D_{x}(\infty) / U \Lambda$ and $\varepsilon_{x}=5.93 u_{*}^{\prime} H=5.93 \sqrt{\tau^{\prime} / \rho} H$ defining the flat bed turbulent mixing coefficient [32], one can finally write

$$
D_{x} \cong \frac{1}{8 \pi^{2}}\left(\frac{\Delta}{H}\right)^{2} \frac{\sqrt{2 / f^{\prime}}}{\left(1+\left(2 / \pi^{2} f^{\prime}\right)\right)} .
$$

As Figure 8 explains, the regime value of the dimensionless longitudinal dispersion coefficient (which practically represents one-half of the time-derivative of the solute cloud inertia moment and is then a measure of the rate at which the cloud is dispersed and diluted by the flow in the given direction) generated by the dune-like periodical nonuniformity is strongly affected by the bed-form steepness, raising with $\Delta / \Lambda$ everything else being the same (more intense 


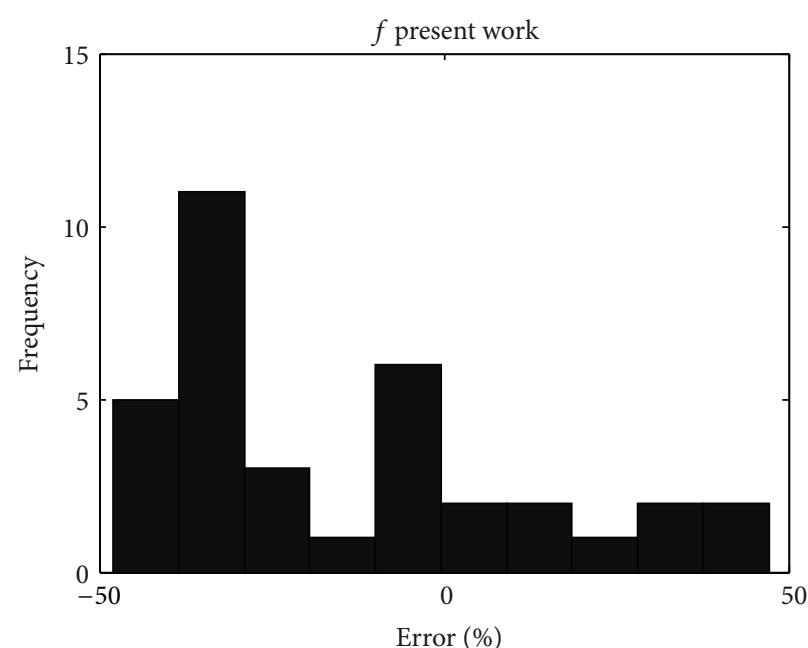

(a)

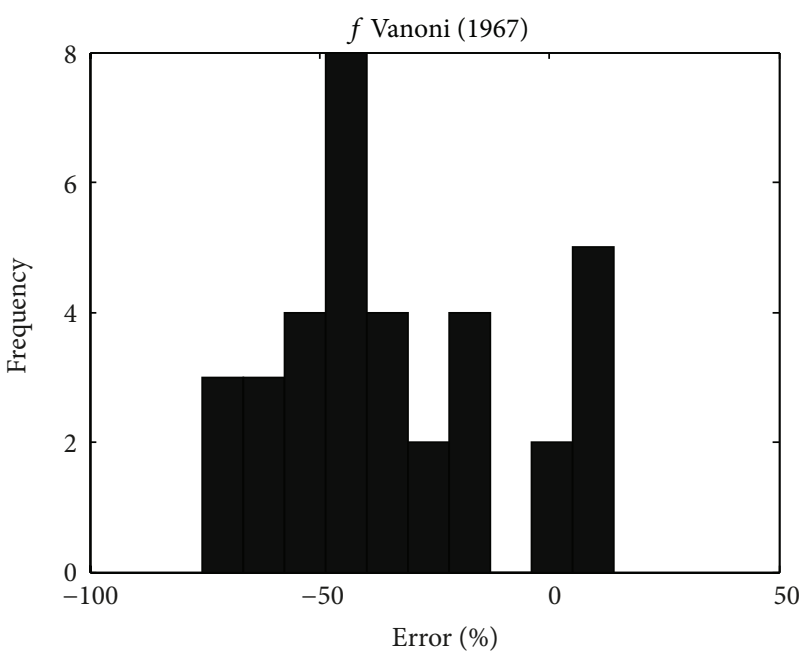

(c)

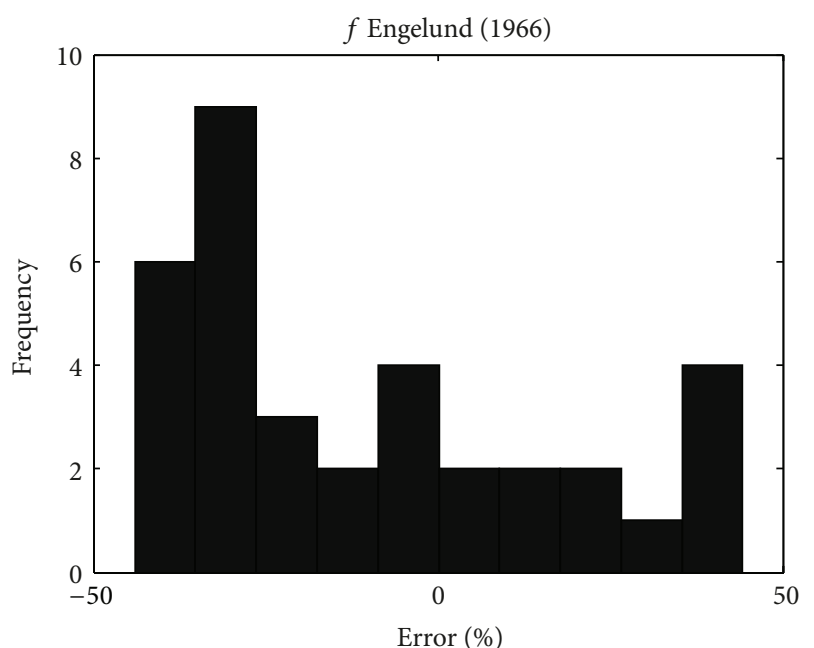

(b)

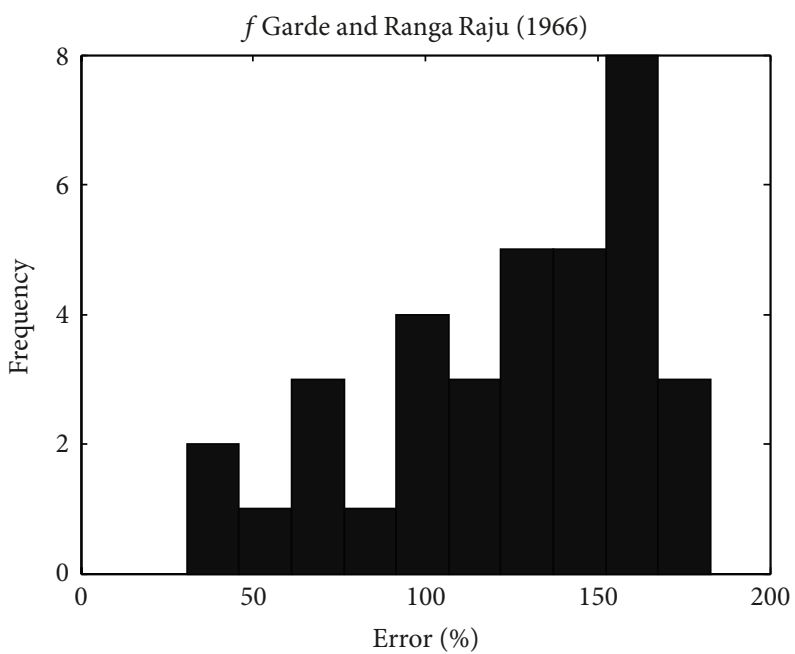

(d)

Figure 4: Histograms of percentage errors corresponding to the use of (10) and (12).

macrovortices dissipation). Furthermore, whereas for low values of $\Delta / \Lambda$ the coefficient is almost independent of the degree of wall turbulence depending on $f^{\prime}$, as $\Delta / \Lambda$ increases the asymptotic $D_{x}$ is more and more influenced by the skin friction factor, to which it is directly related. We could say that, in terms of hydrodynamic dispersion, the inclined planes of upstream and downstream faces of the dune seem to have the same function of the river meanders. Indeed, less rough flow field contours induce less turbulent (and, therefore, less uniform) time-mean velocity profiles due to the slower transverse mixing. Less uniform wall velocity profiles in turn cause a more pronounced solute hydrodynamic dispersion along the local flow direction and, by continuity, a consequent reduced dispersion along the orthogonal ones. If the local flow direction is considerably different from the straight horizontal direction characterizing straight-axis flatbed rivers (highly meandering rivers = horizontal deviations; highly undulated beds = vertical deviations), the longitudinal dispersion diminishes. That would explain why, as $\Delta / \Lambda$ increases, the longitudinal dispersion coefficient becomes more sensitive to the skin roughness variations.

\section{Conclusions}

Present work has proposed the analytical derivation of a simple and easily usable closed-form solution for the estimation of the total flow resistance in presence of river dunes, based on the application of the global equilibrium equations. The final expression accounts for skin and shape roughness in a non trivially additive way and for the free surface undulation as a function of the Froude number; the intrinsically non stationary phenomenon of flow separation at the dune crest is handled in terms of resistance based an equivalent steady flow configuration. 


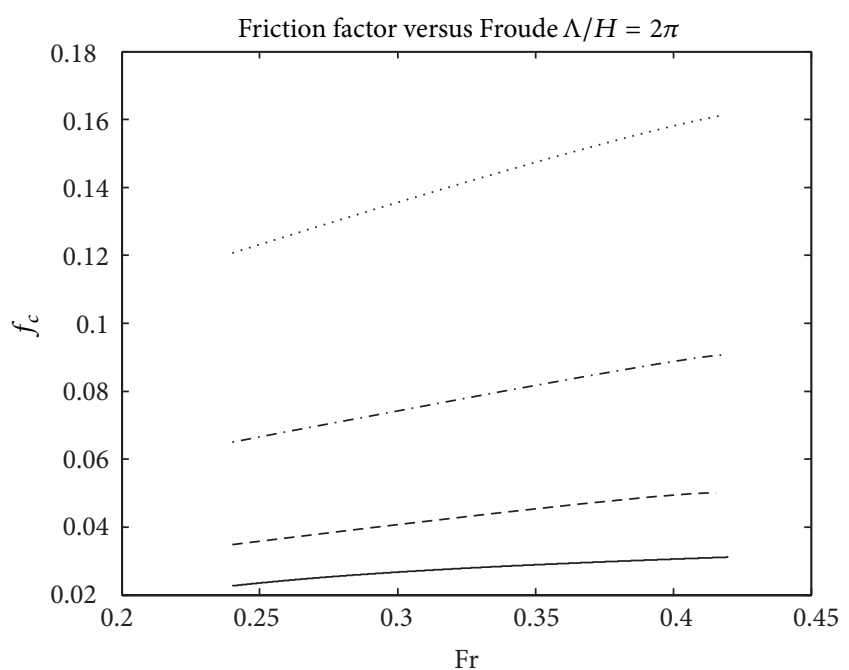

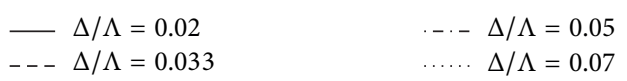

FIGURE 5: Behaviour of the calculated friction factor as a function of Froude number.

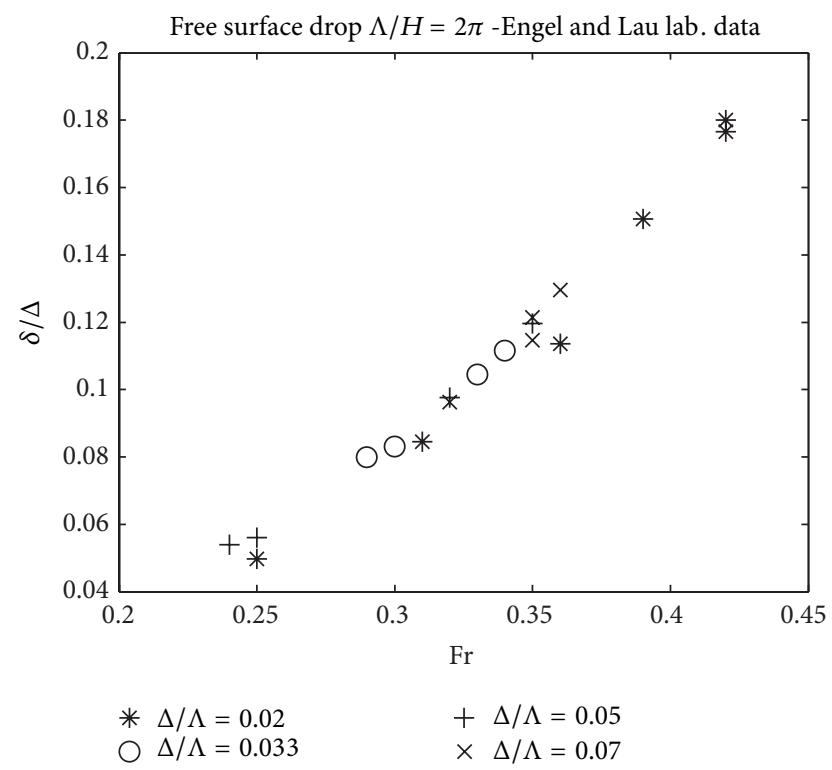

FIGURE 6: Effect of Froude number on the free surface relative drop.

That expression is tested by comparing the analytical estimates of the total friction factor with the laboratory measurements provided by Engel and Lau [9], against the equations proposed by classical literature works [5-7]. In the sampled conditions (for varying average flow velocity and everything else being the same), the three classical formulas rewritten in terms of dimensionless friction factor (12) are not able to account for the effect of the free surface undulation and would provide a constant value for $f$ regardless of the Froude number. Conversely, (10), as shown by Figures 2 and 3 , seems to be able to capture the influence systematic and separated of that crucial parameter by providing friction

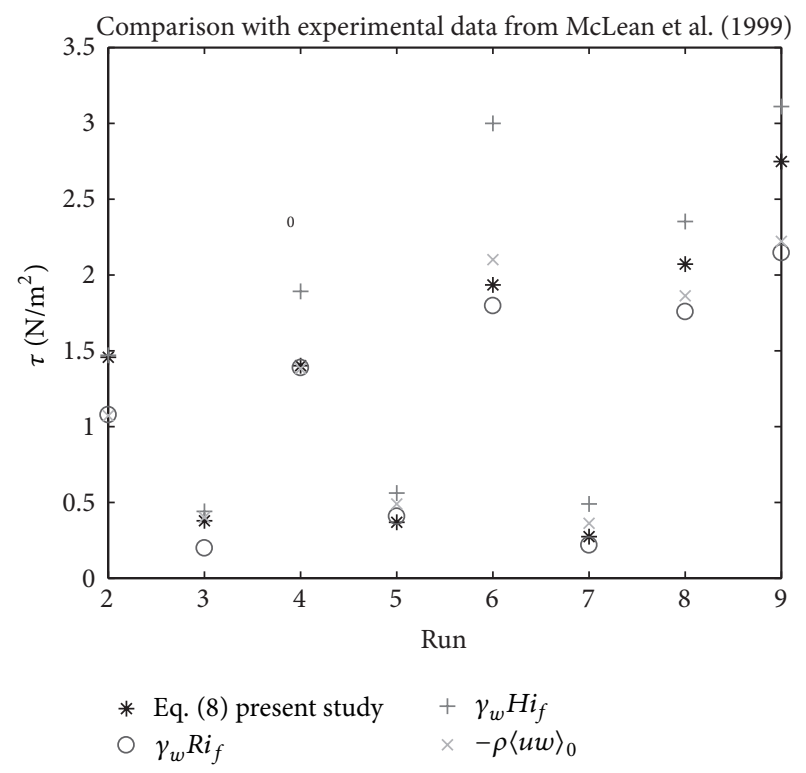

Figure 7: Comparison between measured [15] and calculated total shear stresses.

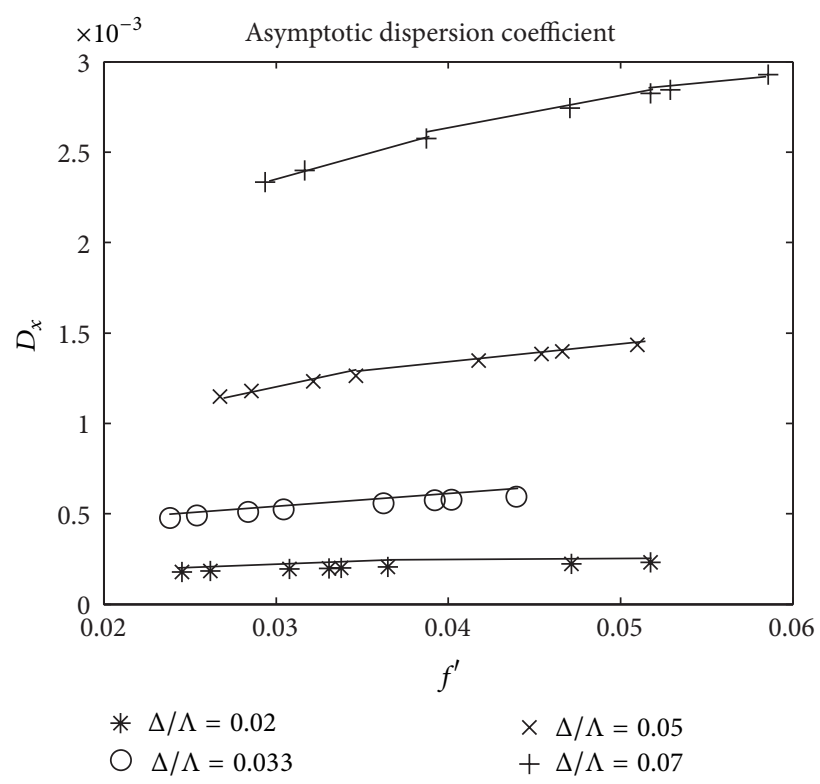

FIgURE 8: Behaviour of the dimensionless asymptotic dispersion coefficient as a function of the skin friction factor for different dune slopes.

factor values rather close to the measured ones, especially for the mildly steep case $\Delta / \Lambda=0.05$. On the other hand, the comparison of the performances provided by the 4 equations (Figure 4) in terms of percentage error histograms reveals the considerable interpretative improvement of the phenomenon represented by (10). Figure 5 highlights a definite increase of the flow resistance with the Froude number that is more pronounced in presence of steeper bedforms, as one would expect due to the more intense disturbances induced within the core of the turbulent flow. The analysis of the relative free 
surface drop in Figure 6 shows the substantial independence of that parameter on the dune slope and its monotonic increase with the Froude number. A further development of the research could address the comprehension and the mathematical modelling of the mechanisms through which the skin roughness resistance is modulated when added to the shape resistance, not only as a function of the geometric characteristics of the sand waves sequence but also as a consequence of the effective cross-sectional velocity profiles, which should be reasonably different from the flat bed logarithmic functions constituting the outcome of the classic Prandtl theory and which justify equations like (11).

In order to double-check the soundness of the proposed approach, (8) was tested based on the experimental measurements presented by McLean et al. [15] and including the total shear stress estimated from the flow dept, the total shear stress estimated from the hydraulic ratio, and the turbulent Reynolds shear stress detected at the bottom. Although one half of the runs was performed with a quite high value of dune slope (0.1) and, therefore, theoretically in contrast with the hypotheses characterizing the proposed approach, the shear stress estimates obtained by (8) fall well inside the range of $\tau$ values provided by the experimental measurements or, at the most, coincide with one/two of them, demonstrating the robustness of our analytical approach even in quite extreme conditions.

Finally, when analyzed in terms of hydrodynamic dispersive properties as a function of the skin roughness on the basis of a previously derived analytical solution, the dune-covered beds seem to behave like meandering channels, responsible for a globally enhanced hydrodynamic solute particles spreading, though with a relatively reduced effect in presence of a less pronounced riverbed modelling.

\section{References}

[1] H. A. Einstein, The Bed-Load Function for Sediment Transportation in Open Channel Flows, U.S. Department of Agriculture, Soil Conservation Service, Washington, DC, USA, 1950.

[2] H. A. Einstein and N. L. Barbarossa, "River channel roughness," Transactions of the American Society of Civil Engineers, vol. 117, pp. 1121-1146, 1952.

[3] H. K. Liu and S. Y. Hwang, "Discharge formula for straight alluvial channels," Journal of the Hydraulic Division, vol. 85, article HY11, 1959.

[4] M. S. Yalin, "Geometrical properties of sand waves," Journal of Hydraulic Engineering, vol. 90, article HY5, pp. 105-120, 1964.

[5] F. Engelund, "Hydraulic resistance of alluvial streams," Journal of the Hydraulic Division, vol. 92, article NY2, pp. 315-326, 1966.

[6] R. J. Garde and K. G. Ranga Raju, "Resistance relationship for alluvial channel flow," Journal of the Hydraulic Division, vol. 94, article HY4, 1966.

[7] V. A. Vanoni and L. S. Hwang, "Relation between bed forms and friction in streams," Journal of the Hydraulic Division, vol. 93, article HY3, pp. 121-144, 1967.

[8] C. G. Ilo, "Resistance to flow in alluvial channels," Journal of Hydraulic Engineering, vol. 101, article HY6, pp. 665-679, 1975.

[9] P. Engel and Y. L. Lau, "Friction factor for two-dimensional dune roughness," Journal of Hydraulic Research, vol. 18, no. 3, pp. 213-225, 1980.
[10] M. I. Haque and K. Mahmood, "Analytical study on steepness of ripples and dunes," Journal of Hydraulic Engineering, vol. 112, no. 3, pp. 229-236, 1986.

[11] C. Colosimo, V. A. Copertino, and M. Veltri, "Friction factor evaluation in gravel-bed rivers," Journal of Hydraulic Engineering, vol. 114, no. 8, pp. 861-876, 1988.

[12] H. W. Shen, H. M. Fehlman, and C. Mendoza, "Bed form resistances in open channel flows," Journal of Hydraulic Engineering, vol. 116, no. 6, pp. 799-815, 1990.

[13] S. R. McLean, J. M. Nelson, and S. R. Wolfe, "Turbulence structure over two-dimensional bed forms: implications for sediment transport," Journal of Geophysical Research, vol. 99, no. 6, pp. 727-747, 1994.

[14] T. B. Maddux, S. R. McLean, and J. M. Nelson, "Turbulent flow over three-dimensional dunes: 2 . Fluid and bed stresses," Journal of Geophysical Research, vol. 108, no. 1, 2003.

[15] S. R. McLean, S. R. Wolfe, and J. M. Nelson, "Spatially averaged flow over a wavy boundary revisited," Journal of Geophysical Research C, vol. 104, no. 7, pp. 15743-15753, 1999.

[16] S. R. McLean, V. I. Nikora, and S. E. Coleman, "Doubleaveraged velocity profiles over fixed dune shapes," Acta Geophysica, vol. 56, no. 3, pp. 669-697, 2008.

[17] G. H. Matthes, "Macroturbulence in natural stream flows," Transactions, American Geophysical Union, vol. 28, no. 2, pp. 255-265, 1947.

[18] M. A. Velikanov, Dynamics of Alluvial Streams, vol. 2, Sediment and bed flow, State Publishing House for Theoretical and Technical Literature, Moscow, Russia, 1955.

[19] K. V. Grishanin, Dynamics of Alluvial Streams, Gidrometeoizdat, Leningrad, Russia, 1979.

[20] N. Kondratiev, I. Popov, and B. Snishchenko, Foundations of Hydromorphological Theory of Fluvial Processes, Gidrometeoizdat, Leningrad, Russia, 1982.

[21] M. A. Dementiev, "Investigation of flow velocities fluctuations and their influences on the flow rate of mountainous rivers," Technical Report of the State Hydrogeological Inst. (GGI), vol. 98, 1962.

[22] S. Yokosi, "The structure of river turbulence," Bulletin of the Disaster Prevention Research Institute, Kyoto University, vol. 17, no. 12, 1967.

[23] A. B. Klaven, "Investigation of structure of turbulent streams," Technical Report of the State Hydrogeological Inst. (GGI), vol. 136, 1966.

[24] A. B. Klaven and Z. D. Kopaliani, "Laboratory investigations of the kinematic structure of turbulent flow past a very rough bed," Technical Report of the State Hydrogeological Inst. (GGI), vol. 209, 1973.

[25] R. G. Jackson, "Sedimentological and fluid-dynamic implications of the turbulent bursting phenomenon in geophysical flows," Journal of Fluid Mechanics, vol. 77, no. 3, pp. 531-560, 1976.

[26] M. S. Yalin, River Mechanics, Pergamon Press, Oxford, UK, 1992.

[27] M. S. Yalin and A. M. Ferreira da Silva, Fluvial Processes, IAHR, Deft, 2001.

[28] A. Robert, "Statistical properties of sediment bed profiles in alluvial channels," Mathematical Geology, vol. 20, no. 3, pp. 205225, 1988.

[29] S.-G. Li, L. Venkataraman, and D. McLaughlin, "Stochastic theory for irregular stream modeling. Part I: flow resistance," Journal of Hydraulic Engineering, vol. 118, no. 8, pp. 1079-1090, 1992. 
[30] S.-G. Li and X. Zhou, "Stochastic theory for irregular stream modeling. II: solute transport," Journal of Hydraulic Engineering, vol. 123, no. 7, pp. 610-616, 1997.

[31] M. Pannone, "Transient hydrodynamic dispersion in rough open channels: theoretical analysis of bed-form effects," Journal of Hydraulic Engineering, vol. 136, no. 3, pp. 155-164, 2010.

[32] J. W. Elder, "The dispersion of marked fluid in turbulent shear flow," Journal of Fluid Mechanics, vol. 5, pp. 544-560, 1959. 


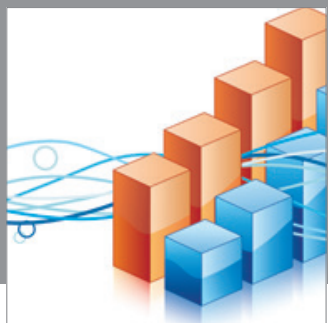

Advances in

Operations Research

mansans

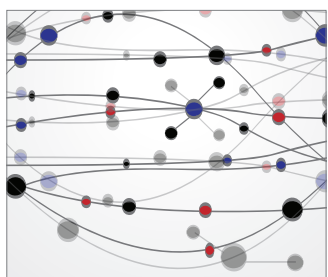

The Scientific World Journal
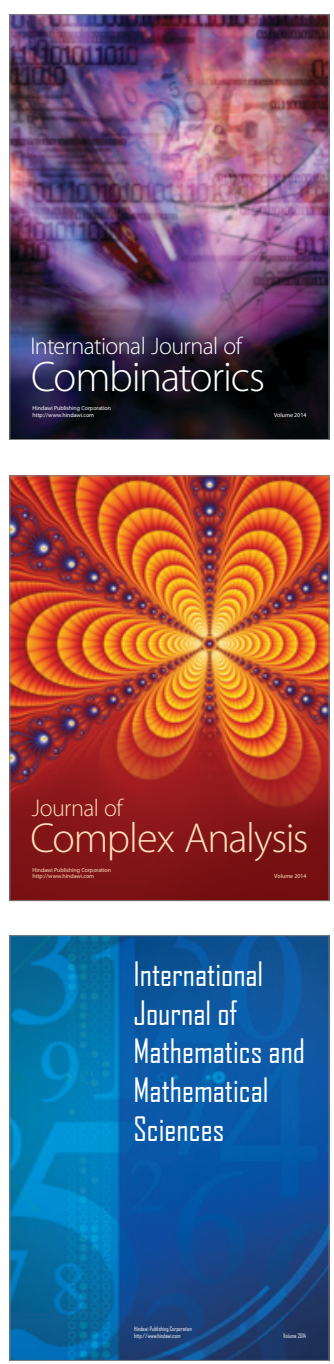
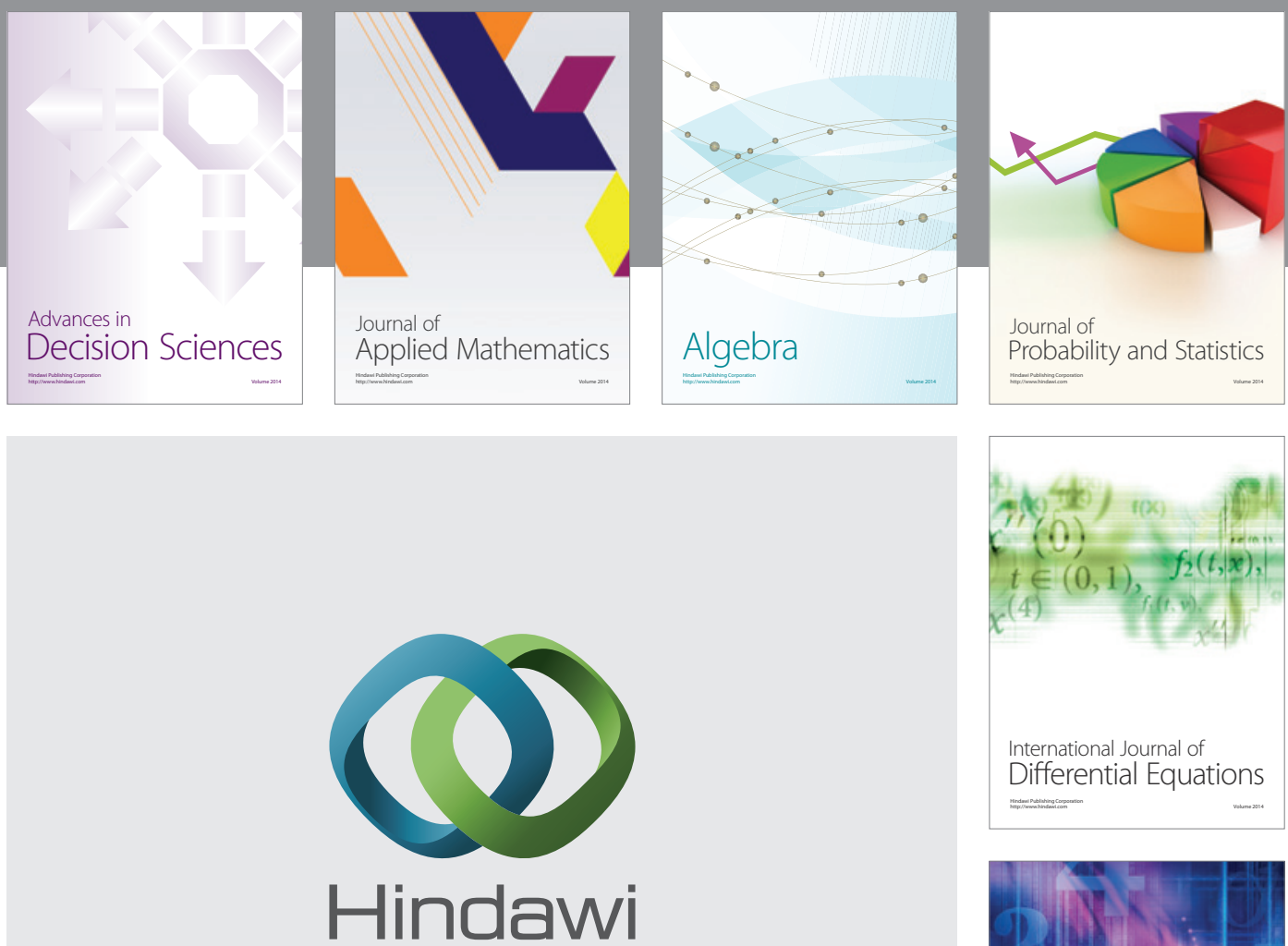

Submit your manuscripts at http://www.hindawi.com
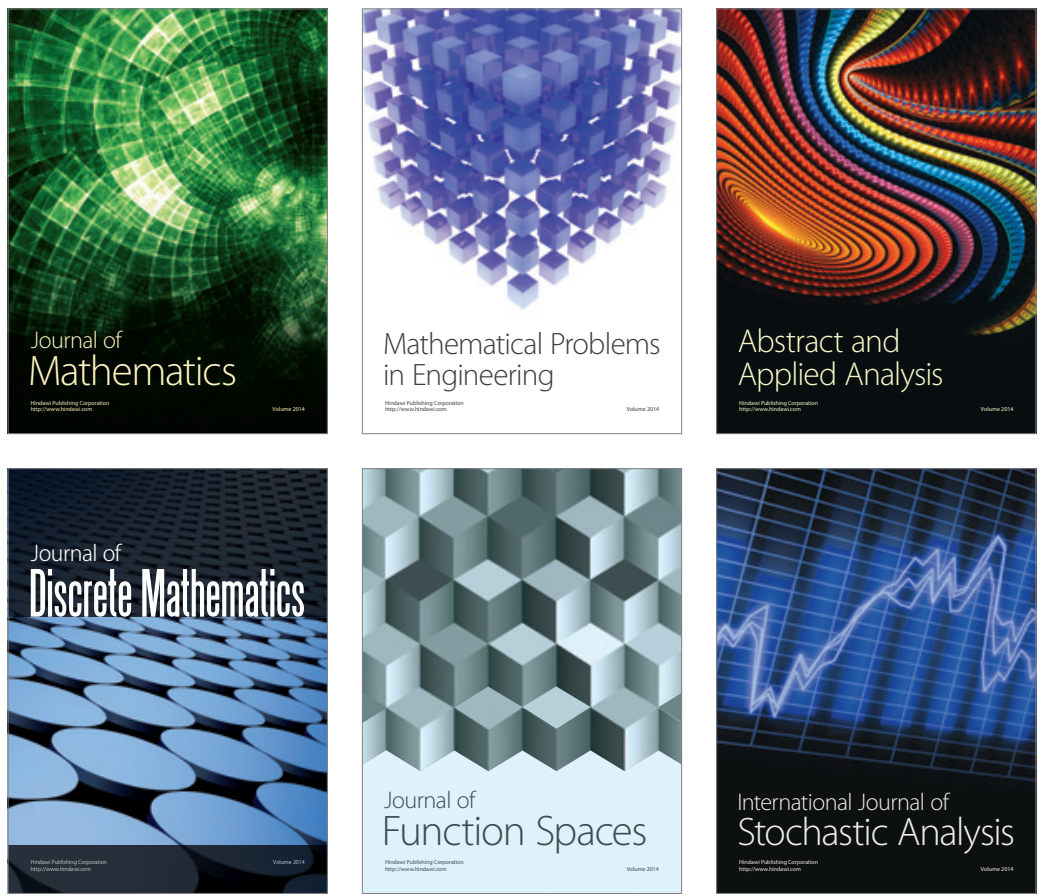

Journal of

Function Spaces

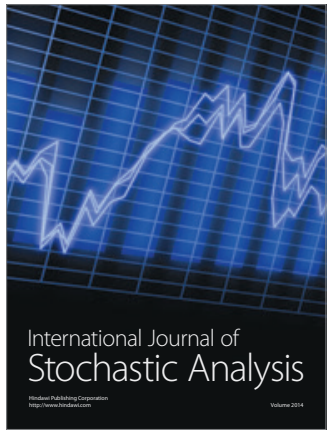

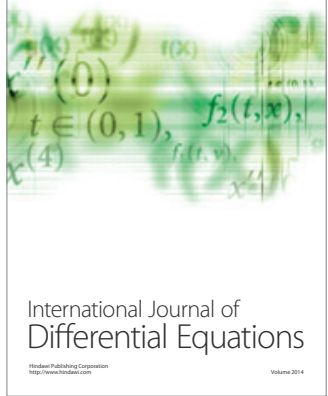
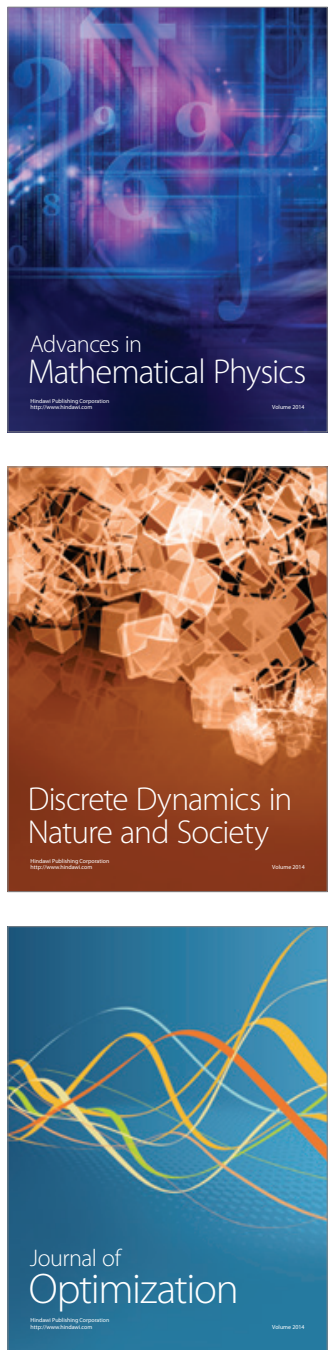